\title{
Acute Exercise Improves Prefrontal Cortex but not Hippocampal Function in Healthy Adults
}

Julia C. Basso, Andrea Shang, Meredith Elman, Ryan Karmouta, AND Wendy A. Suzuki

New York University, Center for Neural Science, New York, New York

(Received April 2, 2015; Final Revision September 30, 2015; AcCePted October 5, 2015)

\begin{abstract}
The effects of acute aerobic exercise on cognitive functions in humans have been the subject of much investigation; however, these studies are limited by several factors, including a lack of randomized controlled designs, focus on only a single cognitive function, and testing during or shortly after exercise. Using a randomized controlled design, the present study asked how a single bout of aerobic exercise affects a range of frontal- and medial temporal lobe-dependent cognitive functions and how long these effects last. We randomly assigned 85 subjects to either a vigorous intensity acute aerobic exercise group or a video watching control group. All subjects completed a battery of cognitive tasks both before and 30, 60, 90, or 120 min after the intervention. This battery included the Hopkins Verbal Learning Test-Revised, the Modified Benton Visual Retention Test, the Stroop Color and Word Test, the Symbol Digit Modalities Test, the Digit Span Test, the Trail Making Test, and the Controlled Oral Word Association Test. Based on these measures, composite scores were formed to independently assess prefrontal cortex- and hippocampal-dependent cognition. A three-way mixed Analysis of Variance was used to determine whether differences existed between groups in the change in cognitive function from pre- to post-intervention testing. Acute exercise improved prefrontal cortex- but not hippocampal-dependent functioning, with no differences found between delay groups. Vigorous acute aerobic exercise has beneficial effects on prefrontal cortex-dependent cognition and these effects can last for up to $2 \mathrm{hr}$ after exercise. (JINS, 2015, 21, 791-801)
\end{abstract}

Keywords: Learning, Memory, Cognition, Prefrontal cortex, Hippocampus, Plasticity

\section{INTRODUCTION}

Wheel running in rodents, a model for physical activity in humans, produces a variety of morphological, neurochemical, and electrophysiological alterations in various brain regions (Boecker, Hillman, Scheef, \& Struder, 2012; Greenwood \& Fleshner, 2011; Meeusen, 2005; Voss, Vivar, Kramer, \& van Praag, 2013). These changes are thought to underlie the behavioral improvements caused by exercise, especially those in learning and memory (Voss et al., 2013). Similarly, evidence is emerging that in humans, physical activity or enhanced physical fitness promotes morphological brain changes and improves learning, memory and cognition. For example, magnetic resonance imaging (MRI) studies demonstrate that long-term exercise causes growth in several brain regions, including the prefrontal cortex, hippocampus, and striatum (Chaddock, Erickson, Prakash, Kim, et al., 2010; Chaddock, Erickson, Prakash, VanPatter et al., 2010;

Correspondence and reprint requests to: Wendy A. Suzuki, New York University, Center for Neural Science, 4 Washington Place, Room 809, New York, NY 10003. E-mail: ws21@ nyu.edu
Colcombe et al., 2006; Erickson et al., 2011; Verstynen et al., 2012). Additionally, compared to their low-fit counterparts, high-fit individuals show greater levels of cognitive functioning, especially in prefrontal cortex-dependent tasks (Guiney \& Machado, 2013).

Although the majority of rodent research has used chronic exercise paradigms, studies of acute exercise in humans are common, with the bulk of this work examining the effects on prefrontal cortex-dependent functions, such as information processing, reaction time, attention, crystalized intelligence, executive functioning, and memory (Chang, Labban, Gapin, $\&$ Etnier, 2012). Meta-analyses of the human acute exercise literature report modest positive effects on learning, memory, and cognition, although individual studies report a wide range of effects, from strongly positive to detrimental (Chang et al., 2012; Roig, Nordbrandt, Geertsen, \& Nielsen, 2013; Tomporowski, 2003).

These contradictory results may be due to a wide range of methodological differences and/or weakness of these studies (Chang et al., 2012). For example, while some studies observed significant improvements in cognition with acute exercise using a randomized control design (Coles \& Tomporowski, 2008; 
Hopkins, Davis, Vantieghem, Whalen \& Bucci, 2012; Loprinzi \& Kane, 2015; Netz, Tomer, Axelrad, Argov, \& Inbar, 2007), most have used either a within-subjects design or had no control group (i.e., compared scores before $v s$. after exercise). Additional common problems with previous studies include: being underpowered due to small sample sizes (10 to 20 subjects); using low-intensity workouts or intense workouts of very short duration $(3,6$, or $10 \mathrm{~min})$; examining a limited range of cognitive abilities (mainly tasks requiring prefrontal cortex functioning); and testing cognitive performance too soon after exercise, perhaps before physiological measures returned to baseline.

Here, we implemented a randomized controlled design with 85 subjects assigned to either an acute exercise or a video watching control group, with cognitive testing between $30 \mathrm{~min}$ and $2 \mathrm{hr}$ after exercise. Video watching was chosen as a control due to its non-physical but engaging nature, and because it has been used in previous acute exercise studies (Coles \& Tomporowski, 2008; Ellemberg \& St-LouisDeschenes, 2010; Emery, Honn, Frid, Lebowitz, \& Diaz, 2001). For the acute exercise group, we chose a vigorous level of exercise with subjects attempting to achieve $85 \%$ of their maximum heart rate (MHR) during a 50-min exercise session (with additional 5-min warm up and cool down periods). This intensity was chosen based on previous metaanalyses showing that moderate- to vigorous-intensity acute exercise protocols are the most beneficial for cognitive improvement (Chang et al., 2012). Participants completed neuropsychological tests designed to tap a wide range of frontal- and medial temporal lobe-dependent functions before and after the hour-long bout of either aerobic exercise or video watching.

We hypothesized that acute aerobic exercise would improve prefrontal cortex-dependent cognitive functioning in healthy adults, with effects lasting up to $2 \mathrm{hr}$. We chose this 2-hr period as studies in rodents indicate that acute exercise increases both neurotransmitter and neurotrophin content in the $2 \mathrm{hr}$ after exercise cessation (Meeusen, Piacentini, \& De Meileir, 2001; Rasmussen et al., 2009), and we hypothesized that these physiological changes may contribute to exercise-induced enhancements in cognition. Additionally, we hypothesized that hippocampal-dependent cognition may show either no change or a less robust change from pre- to post-intervention sessions than prefrontal cortex-dependent cognition because the former may require brain changes that result from chronic exercise, as can be seen in the case of cognitive improvements in rodents due to running-induced neurogenesis, which requires several consecutive days of running (Kronenberg et al., 2006).

\section{METHODS}

\section{Participants}

Ninety-two subjects were recruited from the New York University community and surrounding area. Subjects were recruited through flyers placed around campus. Eighty-five subjects (51 female; 34 male), aged 18-35 (mean $=22.21$; \pm 0.45 SEM), completed the study. Subjects were excluded if they: had undergone major surgery within the prior 6 months, had a past or present history of drug or alcohol abuse, had a diagnosed psychiatric or neurological condition, were taking medication known to affect cognition, or were unable to safely participate in an aerobic exercise program. All subjects gave their informed consent before the study. Informed consent, all study documents, and all data included in this manuscript were approved by and in compliance with the New York University Committee on Activities Involving Human Subjects.

\section{Procedure}

Subjects were randomly assigned to a video watching group $(n=42)$ or an aerobic exercise group $(n=43)$ and randomly assigned to conduct post-intervention cognitive testing 30 (Exercise $n=11$; Control $n=10$ ), 60 (Exercise $n=11$; Control $n=10$ ), 90 (Exercise $n=10$; Control $n=10$ ), or $120 \mathrm{~min}$ (Exercise $n=11$; Control $n=12$ ) after video watching or exercise (Figure 1). Subjects were asked to refrain from consuming food $2 \mathrm{hr}$ before testing and any caffeinated products $4 \mathrm{hr}$ before testing. All testing sessions occurred between 9:00 a.m. and 7:00 p.m., and depending on the delay period, the entire testing session took from 3 to $5 \mathrm{hr}$.

\section{Video watching subjects (control group)}

For our control group, we wanted an experience that was stimulating and engaging without involving physical activity or producing a significant increase in heart rate. Therefore, control subjects engaged in a 60-min video watching session of an episode of the television series 24. Previous studies using video watching as a control intervention used educational documentaries, children's television shows, and

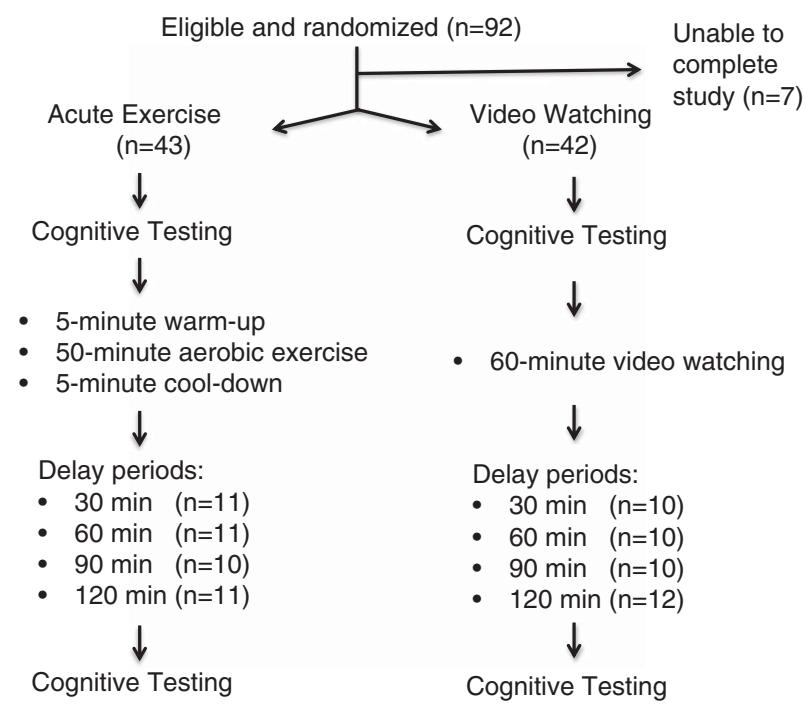

Fig. 1. Flow chart of study design. 
videos describing the beneficial effects of exercise (Coles \& Tomporowski, 2008; Ellemberg \& St-Louis-Deschenes, 2010; Emery et al., 2001). We chose 24 because of the exciting and suspenseful nature of this show. Subjects were informed to pay attention to the show, and research assistants made periodic visual checks to ensure that subjects were actively watching.

\section{Exercise subjects}

Exercise subjects engaged in a 50-min vigorous-intensity physical activity session on a stationary bicycle with a 5-min warm up and 5-min cool down period for a total of $60 \mathrm{~min}$ of exercise. Heart rate was measured using a chest and wristbased monitor (Polar FT7). MHR was calculated for each subject (220-age). Subjects were instructed that their target heart rate was $85 \%$ of MHR, and that they should try to maintain this heart rate throughout as much of the exercise session as possible. Subjects were asked to step onto a stationary bicycle (LifeFitness, model LSTV-0201-01) and begin pedaling. The first $5 \mathrm{~min}$ consisted of a warm-up, followed by $50 \mathrm{~min}$ of vigorous-intensity aerobic exercise, and finally a 5-min cool-down. Heart rate was recorded starting at time zero and then once every $5 \mathrm{~min}$, and the time at which subjects reached their target heart rate was also recorded. To confirm that subjects returned to their resting heart rate (RHR) by the time of their post-intervention cognitive testing, 30 min was chosen as the shortest delay period and heart rate was monitored every 15 min during this post-intervention time period. RHR was taken as the lowest heart rate established during the entire testing period, either before or after exercise. For all exercise subjects, weight and height were recorded and body mass index (BMI) was calculated [mass $(\mathrm{kg}) /$ height $(\mathrm{m})^{2}$ ].

\section{Delay period}

During the delay period subjects were free to do anything except sleep, eat, or exercise. Delay period activities included reading, completing homework assignments, making phone calls, or using the Internet. Subjects who were assigned to a delay period of $1 \mathrm{hr}$ or longer were provided with a granola bar.

\section{Neuropsychological Tests}

All neuropsychological tasks were conducted both before and after aerobic exercise or video watching interventions. Paper and pencil versions were administered for all tests except the Modified Benton Visual Retention Task (MBVRT), which was administered on a computer. Persons administering the neuropsychological tasks were extensively trained and gave all tasks in accordance with standardized procedures. Considering the minimal amount of time between pre- and post-intervention testing, alternate versions of the tasks were used when available [i.e., for the Hopkins Verbal Learning Test-Revised (HVLT-R) and the Controlled Oral Word Association Test]. The order of administration was fixed to ensure that enough time elapsed between the immediate and delayed versions of both the HVLT-R and the MBVRT. To ensure that subjects were engaged in each task, they were visually monitored during all testing procedures.

For this study, we were interested in examining the effects of acute exercise on both prefrontal cortex- and hippocampaldependent cognition. We, therefore, targeted a wide range of standardized, well-validated prefrontal cortex-dependent tasks and select medial temporal lobe-dependent tasks. For all tasks, raw scores were converted to $t$ scores, $Z$ scores, or scaled scores based on age and/or education. Tests were presented in the following order. All raw data are presented in Supplementary Table 1.

\section{Hopkins Verbal Learning Test Revised (HVLT-R)}

This verbal learning and memory task taps the functioning of the prefrontal cortex and hippocampus (delayed and recognition component only) (Brandt \& Benedict, 2001). Subjects were read a list of 12 words and asked to immediately recall as many words as possible on three distinct, consecutive trials (total recall score) and again $20 \mathrm{~min}$ later (delayed recall score). The retention score was calculated by dividing the delayed recall score by the greatest number of words remembered in learning trials two or three. In the recognition component, the experimenter read 24 words (half targets and half lures), and subjects were ask to identify which words had been presented previously. The recognition discrimination index was calculated by subtracting the total number of false positives from the total number of true positives. Raw scores were transformed to $t$ scores based on age and the version of the task used (Brandt \& Benedict, 2001), using the normative values with the closest age midpoint to the subject's age.

\section{Modified Benton Visual Retention Test (MBVRT)}

This is an object recognition memory paradigm that tests the functioning of the dentate gyrus of the hippocampus (Brickman, Stern, \& Small, 2011). Subjects were shown a complex geometric image for $10 \mathrm{~s}$ and then asked to select the image they just saw from an array of four images (10 trials). The Benton recognition score was the total number of correctly identified images. During a matching trial, subjects were shown a complex geometric image for $10 \mathrm{~s}$ and then asked to select the image they just saw from an array of two images (41 trials) (matching score). During a delayed recognition trial, subjects were shown a complex geometric image and asked whether or not this was one of the images they had seen earlier (82 trials) (delayed score). No normative values existed for this test and so to calculate $t$ scores, all raw scores were compared to the average of the pre-intervention values for the recognition score, matching score, and delayed score respectively.

\section{Stroop Color and Word Test}

This is a prefrontal cortex-dependent task of attention and response inhibition (Golden, 1975). For the word 
component, subjects were instructed to read the words "RED", "GREEN", and "BLUE" all in black as fast as they could until the experimenter said to stop (word score). For the color component, subjects were instructed to name the colors of "XXXX's" printed in red, green or blue as fast as they could (color score). For the color-word component, subjects were instructed to name the color of the words "RED", "GREEN", and "BLUE" as fast as they could (color-word score). The word and the color never matched (all incongruent). The interference score was calculated by subtracting the predicted color-word score (word score $\times$ color score/ word score + color score) from the actual color-word score. Raw scores were converted to $t$ scores based on age and years of education (Golden, 1978).

\section{Symbol Digit Modalities Test (SDMT)}

This is a prefrontal cortex-dependent task of visuomotor processing (Smith, 1991). Subjects were given a key of symbol-number pairs. They were then asked to fill in as many symbols with their corresponding numbers as possible in $90 \mathrm{~s}$. The SDMT score was the number of correct substitutions. Raw scores were transformed to $Z$ scores based on age and level of education (Centofani, 1975).

\section{Digit Span Test (DST)}

This is a prefrontal cortex-dependent task of attention and working memory (Wechsler, 1981). Subjects were instructed to listen to and then immediately recall a list of random numbers, which increased by one every two trials (digits forward). The test ended when subjects could not remember the complete list of numbers in both trials. For the digits backward component, the instructions were similar except that subjects were instructed to recall the numbers in reverse order. The DST score was the total number of trials completed in both the digits forward and digits backward components. Raw scores were converted to scaled scores based on age (Wechsler, 2008).

\section{Trail Making Test (TMT)}

This test measures prefrontal cortex-dependent attention and cognitive flexibility (Tombaugh, 2004). Subjects were instructed to connect in order as fast as they could 25 randomly placed, encircled numbers on a page (TMT A). For TMT part B, subjects were instructed to connect in alternate alphanumeric order 25 randomly placed, encircled numbers and letters. TMT A and B scores were the total number of seconds it took to complete part A and B respectively. Raw scores were transformed to $Z$ scores based on age (Yeudall, Redden, Gill, \& Stefanyk, 1987; Tombaugh, 2004).

\section{Controlled Oral Word Association Test (COWAT)}

This is a prefrontal cortex-dependent task of verbal fluency (Benton \& Hamsher, 1989). Subjects were given $1 \mathrm{~min}$ to name as many words as they could that began with a different letter of the alphabet. The COWAT score was the total number of words that were generated during three trials. Raw scores were converted to $Z$ scores based on age and years of education (Ruff, Light, Parker, \& Levin, 1996; Tombaugh, Kozak, \& Rees, 1999).

\section{Wechsler Test of Adult Reading (WTAR)}

This reading test was performed only at pre-intervention to ensure that both groups of subjects had equivalent levels of intelligence. Subjects were asked to read aloud each of 50 words with atypical grapheme to phoneme translations. The WTAR score was the total number of words that were pronounced correctly.

\section{Creation of a Composite Score}

Our strategy to examine the effect of acute exercise on prefrontal cortex- and hippocampal-dependent function was to create a composite score for each of these measures. Composite measures are commonly produced based on both the aims of the study as well as the theory behind what particular variables measure (Pennington, Moon, Edgin, Stedron, \& Nadel, 2003). Therefore, we decided to create two composite scores by averaging together $Z$ scores of tasks that tap a core neuropsychological function associated with either the prefrontal cortex or hippocampus (Cutter et al., 1999; Pennington et al., 2003). For the prefrontal cortex, this included working memory, processing speed, verbal fluency and cognitive inhibition. For the hippocampus, this included long-term memory and pattern separation.

\section{Step 1: Z scores}

Because each task had an inherently different scoring system, it was necessary to transform all scores into a common metric. We thus standardized all scores into $Z$ scores whereby we compared each individual score to an average score. Negative and positive $Z$ scores reflect scores that fall below or above the average in standard deviations, respectively. To derive $Z$ scores for each neuropsychological test, unique normative values from large studies were used to represent the population averages and standard deviations. For the MBVRT, no normative values were available and so the mean and standard deviation of all data from the pre-intervention testing sessions were used to calculate $Z$ scores. For certain tests, only $t$ scores or scaled scores were available. If this was the case, a psychometric conversion table was used to convert these scores to $Z$ scores. For the TMT, a higher score represented a decrease in function. Therefore, $Z$ scores were multiplied by -1 so that in all cases higher $Z$ scores corresponded to better functional outcomes.

\section{Step 2: Construction of the composite score}

To obtain an understanding of brain-region specific functioning, $Z$ scores from each component of the neuropsychological tasks were averaged together. For prefrontal 
cortex functioning, $Z$ scores were averaged together for all components of the Stroop Task (Word, Color, Color-Word, and Interference Score), the SDMT score, HVLT-R Total Recall Score, DST Score, and all components of the TMT (TMT A and B Score). For hippocampal functioning, $Z$ scores were averaged together for the HVLT-R Delayed Recall, Retention, and Recognition Discrimination Index, as well as all components of the MBVRT (Benton Recognition, Matching, and Delayed Score). Average rather than summed $Z$ scores were used because each composite score was comprised of different numbers of neuropsychological tasks. We acknowledge that brain function was not directly measured and that these composite scores may reflect coordinated activity from a series of interconnected brain regions beyond just the prefrontal cortex and hippocampus. With this in mind, we refer to these composite scores as measures of prefrontal cortex and hippocampal functioning, with higher overall scores reflecting better average functioning of either of these structures.

\section{Statistical Analysis}

To ensure that all groups were of equal age, education level and intelligence (WTAR score), a two-way analysis of variance (ANOVA) was conducted, with intervention type (television watching vs. exercise) and delay period (30-, 60-, 90-, or 120-min) as the two between-subjects factors. Additionally, a two-way ANOVA was conducted to determine whether composite measures of prefrontal cortex and hippocampal function were similar across all groups at the pre-intervention time point.

A three-way mixed ANOVA, with prefrontal cortex function as the dependent variable, time as the within-subjects factor (pre- vs. post-intervention), and intervention type and delay period as the between-subjects factors, was used to determine whether the intervention, delay period or their interaction affected prefrontal cortex or hippocampal functioning. All pairwise comparisons were performed for statistically significant main effects. An alpha level of 0.05 was used to determine statistical significance; however, Bonferroni corrections were made for comparisons within each simple main effect considered a family of comparisons. Adjusted $p$-values are reported. Additionally, no outliers were present in the data, all data were normally distributed, all data inherently met sphericity, as only two time points were evaluated, and there was homogeneity of variances for both pre- and postintervention prefrontal cortex and hippocampal function.

\section{RESULTS}

Before conducting our primary statistical analyses, using a two-way ANOVA, we confirmed that age $(F(3,77)=0.613$; $p=.608$; partial $\left.\eta^{2}=0.023\right)$, education $(F(3,77)=0.254$; $p=.858 ;$ partial $\left.\eta^{2}=0.010\right)$, and intelligence level $\left(F(3,77)=0.444 ; \quad p=.723 ;\right.$ partial $\left.\eta^{2}=0.017\right)$ were equivalent across all groups (Table 1).

\section{Exercise Intensity}

No differences between groups were found for BMI $\left(F(3,35)=0.552 ; p=.650 ;\right.$ partial $\left.\eta^{2}=0.045\right)$ or $\mathrm{RHR}$ $\left(F(3,36)=1.126 ; p=.351 ;\right.$ partial $\left.\eta^{2}=0.086\right)($ Table 2$)$. Additionally, all groups had similar average heart rates during the warm up $(F(3,36)=0.261 ; p=.853$; partial $\left.\eta^{2}=0.021\right), 50$-min workout $(F(3,36)=1.052 ; p=.381$; partial $\left.\eta^{2}=0.081\right)$, and cool down $(F(3,34)=0.646$; $p=.591$; partial $\left.\eta^{2}=0.054\right)$ (Table 2). Exercise subjects took an average of $10.66( \pm 1.29$ SEM) min to reach $85 \%$ of their MHR; however, three subjects never reached their target heart rate and instead worked out at $76 \%, 72 \%$, and $67 \%$ of their MHR. On average, subjects worked out at $81.55 \%$ $( \pm 0.77$ SEM) of their MHR, with a range of $67-89 \%$ ensuring that subjects were exercising with a vigorousintensity workload. No differences between groups were found for either time to target heart rate $(F(3,33)=1.163$; $p=.338$; partial $\left.\eta^{2}=0.096\right)$ or intensity of the $50-\mathrm{min}$ workout $\left(F(3,36)=0.609 ; p=.614\right.$; partial $\left.\eta^{2}=0.048\right)$. By the start of the cognitive testing period, all exercise subjects returned to their pre-exercise RHR. Due to technical difficulties, heart rate data on two subjects were not collected. Collectively, these data show that all delay groups were of similar health, as assessed by BMI and RHR, and performed at similar levels throughout their workouts.

\section{Acute Exercise Improves Prefrontal Cortex Functioning}

For the composite measure of prefrontal cortex functioning, all groups scored equally at the pre-intervention time point $\left(F(3,77)=0.472 ; p=.703\right.$; partial $\left.\eta^{2}=0.018\right)($ Table 3$)$.

In analyzing change over time, there was not a statistically significant three-way interaction between time, delay period, and intervention type, $F(3,77)=1.481, p=.226$, partial $\eta^{2}=0.055$. However, there was a statistically significant two-way interaction between time and intervention type, $F(1,77)=10.890, p=.001$, partial $\eta^{2}=0.124$. All other two-way interactions were not statistically significant $(p>.05)$.

Statistical significance of a simple main effect was accepted at a Bonferroni-adjusted alpha level of 0.025. There was a statistically significant main effect of time on the exercise group, $F(1,77)=6.613, p=.012$, partial $\eta^{2}=0.079$, but not on the control group, $F(1,77)=0.958, p=.331$, partial $\eta^{2}=0.012$ (Figure 2). Mean prefrontal cortex function was higher in exercise subjects than video watching subjects, with a mean difference of 0.291 detected, 95\% CI [0.066, 0.516], $p=.012$.

\section{No Significant Effect of Acute Exercise on Hippocampal Functioning}

For the composite measures of hippocampal functioning, we first confirmed that all groups scored equally at the pre-intervention time point $(F(3,77)=1.025 ; p=.386$; partial $\left.\eta^{2}=0.038\right)($ Table 3$)$. 
Table 1. Age, education, and intelligence level

\begin{tabular}{|c|c|c|c|c|c|c|c|c|c|c|c|c|}
\hline \multirow[b]{3}{*}{ Measurement } & \multicolumn{10}{|c|}{ Delay period and intervention type } & \multirow{3}{*}{$\mathrm{p}$ Value } & \multirow{3}{*}{$\begin{array}{c}\text { Partia } \\
\eta^{2}\end{array}$} \\
\hline & \multicolumn{2}{|c|}{$30 \mathrm{Min}$} & \multicolumn{2}{|c|}{$60 \mathrm{Min}$} & \multicolumn{2}{|c|}{$90 \mathrm{Min}$} & \multicolumn{2}{|c|}{$120 \mathrm{Min}$} & \multicolumn{2}{|c|}{ Average } & & \\
\hline & Exercise & Video & Exercise & Video & Exercise & Video & Exercise & Video & Exercise & Video & & \\
\hline Age (years) & $20.45( \pm 0.69)$ & 21.10 & $23.64( \pm 1.54)$ & $22.4( \pm 1.50)$ & $22.30( \pm 1.21)$ & $21.90( \pm 1.34)$ & $21.82( \pm 0.84)$ & $23.83( \pm 1.56)$ & $22.05( \pm 0.57)$ & $22.38( \pm 0.71)$ & .608 & 0.023 \\
\hline Education (years) & $14.18( \pm 0.42)$ & 14.40 & $15.82( \pm 0.54)$ & $15.00( \pm 0.33)$ & $15.30( \pm 0.68)$ & $14.90( \pm 0.59)$ & $15.00( \pm 0.70)$ & $14.67( \pm 0.66)$ & $15.07( \pm 0.30)$ & $14.74( \pm 0.29)$ & .858 & 0.010 \\
\hline WTAR (raw score) & $40.55( \pm 1.29)$ & $41.80( \pm 1.36)$ & $43.55( \pm 1.12)$ & $42.3( \pm 1.45)$ & $40.30( \pm 2.56)$ & $40.60( \pm 1.17)$ & $42.64( \pm 1.92)$ & $40.58( \pm 1.50)$ & $41.79( \pm 0.88)$ & $41.29( \pm 0.68)$ & .723 & 0.017 \\
\hline
\end{tabular}

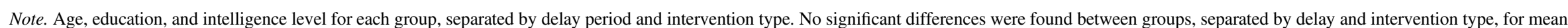
$\left( \pm\right.$ SEM) age (years), education (years), and Wechsler Test of Adult Reading (raw score). The $p$ values and partial $\eta^{2}$ presented represent delay by intervention type interactions.

Table 2. Physiological data

\begin{tabular}{|c|c|c|c|c|c|c|c|}
\hline \multirow[b]{2}{*}{ Physiological measurement } & \multicolumn{5}{|c|}{ Delay period } & \multirow[b]{2}{*}{$\mathrm{p}$ Value } & \multirow[b]{2}{*}{ Partial $\eta^{2}$} \\
\hline & 30 Min & $60 \mathrm{Min}$ & $90 \mathrm{Min}$ & $120 \mathrm{Min}$ & Average & & \\
\hline Body mass index $\left(\mathrm{kg} / \mathrm{m}^{2}\right)$ & $23.63( \pm 0.91)$ & $22.87( \pm 0.74)$ & $22.36( \pm 0.72)$ & $22.49( \pm 0.60)$ & $22.86( \pm 0.37)$ & .650 & 0.045 \\
\hline Resting heart rate (bpm) & $80.27( \pm 2.16)$ & $82.45( \pm 2.75)$ & $76.63( \pm 3.85)$ & $76.60( \pm 2.32)$ & $79.23( \pm 137)$ & .351 & 0.086 \\
\hline Heart rate during warm up (bpm) & $127.91( \pm 7.53)$ & $122.27( \pm 5.57)$ & $124.13( \pm 8.49)$ & $120.20( \pm 4.73)$ & $123.68( \pm 3.21)$ & .853 & 0.021 \\
\hline Heart rate during workout (bpm) & $166.21( \pm 2.61)$ & $158.84( \pm 3.76)$ & $159.56( \pm 4.20)$ & $160.16( \pm 3.18)$ & $161.34( \pm 1.71)$ & .381 & 0.081 \\
\hline Heart rate during cool down (bpm) & $135.64( \pm 4.83)$ & $132.33( \pm 4.91)$ & $139.63( \pm 4.69)$ & $129.40( \pm 6.30)$ & $134.05( \pm 2.62)$ & .591 & 0.054 \\
\hline Time to target heart rate (minutes) & $9.16( \pm 1.04)$ & $9.53( \pm 1.21)$ & $15.22( \pm 5.45)$ & $9.51( \pm 1.33)$ & $10.66( \pm 1.29)$ & .338 & 0.096 \\
\hline Intensity of 50-minute workout ( $\%$ of MHR) & $83.26( \pm 1.09)$ & $80.84( \pm 1.66)$ & $80.97( \pm 2.18)$ & $80.90( \pm 1.42)$ & $81.55( \pm 0.77)$ & .614 & 0.048 \\
\hline
\end{tabular}

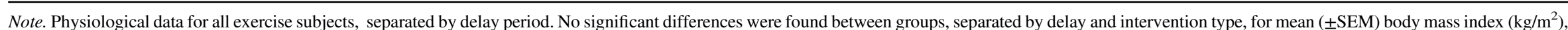

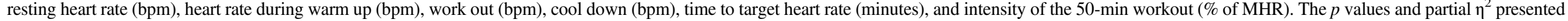
represent delay by intervention type interactions. $\mathrm{kg}=$ kilograms; $\mathrm{m}=$ meters; $\mathrm{BPM}=$ beats per minute; $\mathrm{MHR}=$ Maximum heart rate 

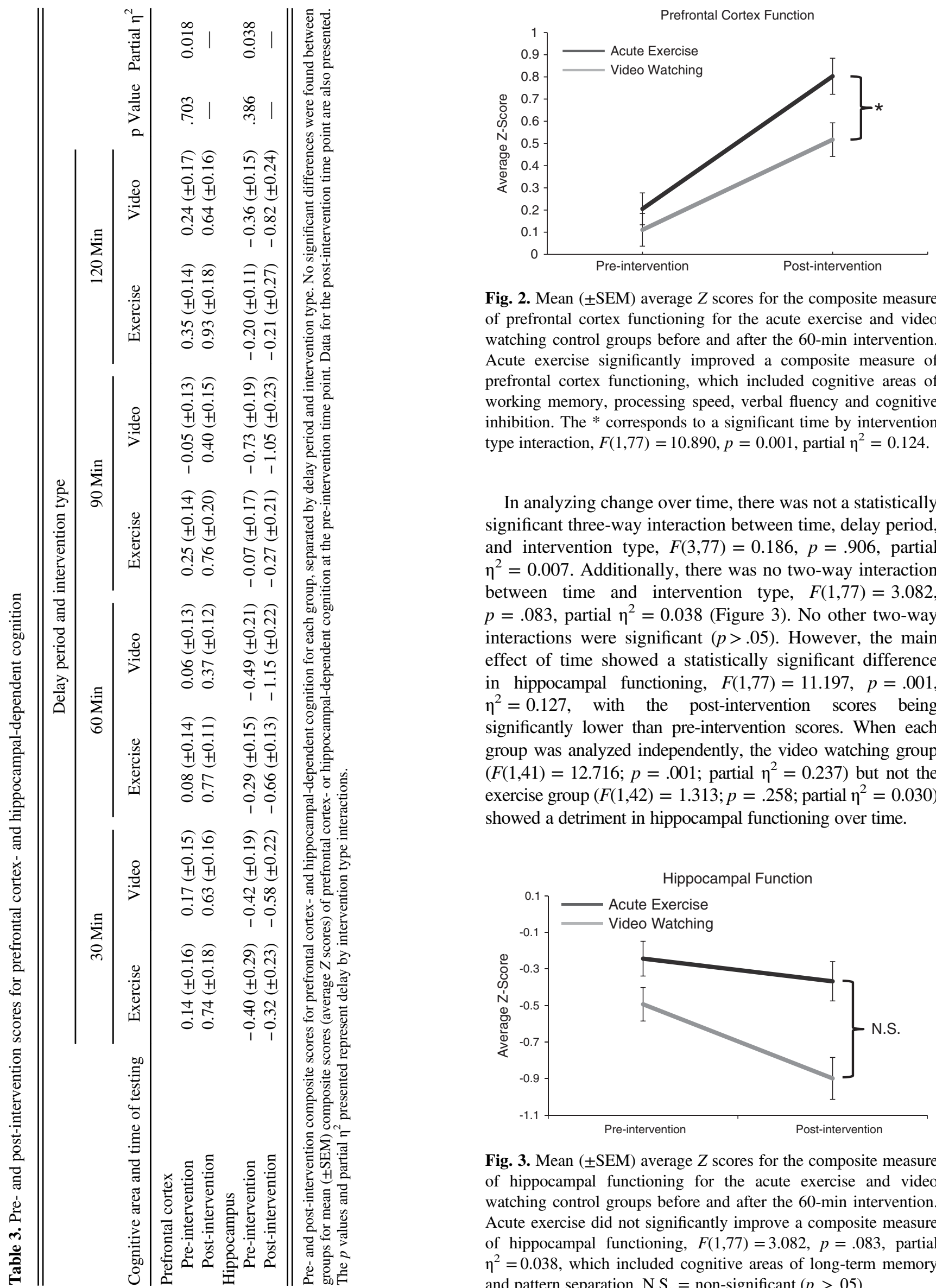

Fig. 2. Mean $( \pm S E M)$ average $Z$ scores for the composite measure of prefrontal cortex functioning for the acute exercise and video watching control groups before and after the 60-min intervention. Acute exercise significantly improved a composite measure of prefrontal cortex functioning, which included cognitive areas of working memory, processing speed, verbal fluency and cognitive inhibition. The $*$ corresponds to a significant time by intervention type interaction, $F(1,77)=10.890, p=0.001$, partial $\eta^{2}=0.124$.

In analyzing change over time, there was not a statistically significant three-way interaction between time, delay period, and intervention type, $F(3,77)=0.186, p=.906$, partial $\eta^{2}=0.007$. Additionally, there was no two-way interaction between time and intervention type, $F(1,77)=3.082$, $p=.083$, partial $\eta^{2}=0.038$ (Figure 3). No other two-way interactions were significant $(p>.05)$. However, the main effect of time showed a statistically significant difference in hippocampal functioning, $F(1,77)=11.197, p=.001$, $\eta^{2}=0.127$, with the post-intervention scores being significantly lower than pre-intervention scores. When each group was analyzed independently, the video watching group $\left(F(1,41)=12.716 ; p=.001 ;\right.$ partial $\left.\eta^{2}=0.237\right)$ but not the exercise group $\left(F(1,42)=1.313 ; p=.258 ;\right.$ partial $\left.\eta^{2}=0.030\right)$ showed a detriment in hippocampal functioning over time.

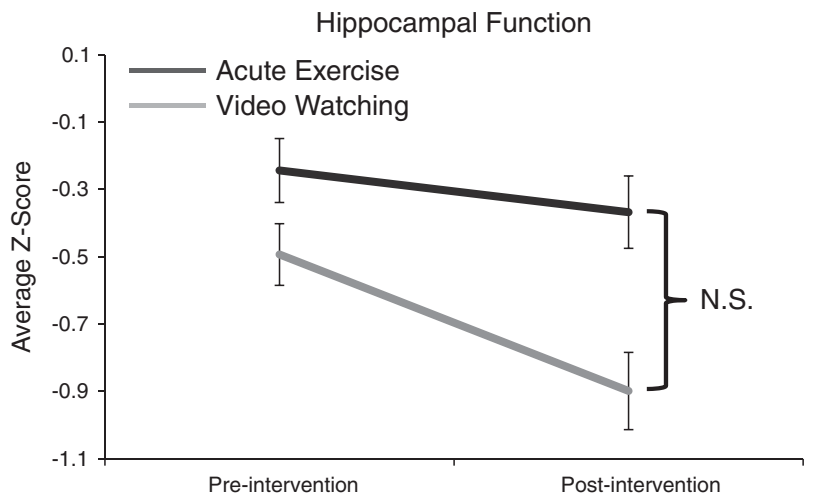

Fig. 3. Mean $( \pm$ SEM) average $Z$ scores for the composite measure of hippocampal functioning for the acute exercise and video watching control groups before and after the 60-min intervention. Acute exercise did not significantly improve a composite measure of hippocampal functioning, $F(1,77)=3.082, p=.083$, partial $\eta^{2}=0.038$, which included cognitive areas of long-term memory and pattern separation. N.S. $=$ non-significant $(p>.05)$. 


\section{DISCUSSION}

The present study used a large battery of neuropsychological tasks to determine whether acute aerobic exercise is capable of improving cognition dependent on the prefrontal cortex or hippocampus. We found that an acute bout of $50 \mathrm{~min}$ of vigorous-intensity aerobic exercise in healthy adults improves prefrontal cortex- but not hippocampal-dependent cognition. We further found that the acute exercise-induced cognitive enhancements lasted longer than previously known, between $30 \mathrm{~min}$ and $2 \mathrm{hr}$ after exercise cessation.

\section{Acute Exercise Improves Prefrontal Cortex Functioning}

The findings from this study indicate that acute aerobic exercise improves executive functioning, a grouping of higher-order cognitive processes that depend on the prefrontal cortex, with a medium to large effect size (7.9\% of the variability in the composite score being explained by the exercise intervention). Executive functions are unique in that they peak rather late in development (i.e., early adulthood) and begin to deteriorate early in the process of healthy aging (Diamond, 2013). Once reaching their peak, executive functions were thought to remain fixed throughout adulthood; however, recent evidence indicates that these cognitive processes may in fact be malleable (Hsu, Novick, \& Jaeggi, 2014). The prefrontal cortex is a brain region that appears particularly sensitive to experience-dependent plasticity throughout life, and this work indicates that aerobic exercise is an intervention that can acutely enhance prefrontal cortex function in early adulthood. Furthermore, this work shows that acute exercise can have beneficial effects even in healthy adults at their theoretical peak of executive functioning.

An extant literature has investigated the effects of acute exercise on cognition and several reviews have been published regarding this work (Etnier et al., 1997; Lambourne \& Tomporowski, 2010; McMorris, 2000; Brisswalter, Collardeau, \& Rene, 2002; Tomporowski \& Ellis, 1986). The discrepant findings in this literature result from the various methodologies utilized in regards to the type of exercise used, the cognitive process measured, and the timing of the post-exercise testing. Some studies used exhaustive, dehydrating exercise, often anaerobic in nature, which produced no or detrimental effects on cognition (Lambourne \& Tomporowski, 2010; Tomporowski, 2003). Our work is most comparable to other studies examining steady-state aerobic exercise on cognition. This research indicates that acute bouts of aerobic exercise improve a variety of cognitive processes including attention, concentration, working memory, reasoning, and planning (Loprinzi \& Kane, 2015; Nanda, Baalde, \& Manjunatha, 2013), although not all prefrontal cortex-dependent tasks are improved by acute aerobic exercise (Nanda et al., 2013; Alves et al., 2014; Wang et al., 2015). Our work adds to this literature by showing that acute aerobic exercise enhances the overall functioning of the prefrontal cortex.
Although executive functions are examined using particular cognitive tasks administered in a laboratory setting, they have been shown to predict a wide range of practical life outcomes such as reading comprehension and mathematics, planning, and problem solving, language processing, selfregulatory behavior, and scholastic achievement (Alloway \& Alloway, 2010; de Jonge \& de Jong, 1996; Duncan et al., 2007; Gathercole, Alloway, Willis, \& Adams, 2006; Hofmann, Schmeichel, \& Baddeley, 2012; Novick, Trueswell, \& Thompson-Schill, 2005; Passolunghi \& Siegel, 2001; Shah \& Miyake, 1999). Therefore, the findings from our work suggest that acute exercise may also beneficially impact real-world functioning, and research in children indicates that this may be true, as children who participate in bouts of acute exercise through physical education classes perform better in tests of mathematical ability (Gabbard \& Barton, 1979; Hillman, Kamijo, \& Scudder, 2011; Howie, Schatz, \& Pate, 2015; McNaughten \& Gabbard, 1993). However, further research is needed to examine whether similar acute exercise interventions affect real world behaviors in healthy adults.

\section{Acute Exercise Does Not Improve Hippocampal Functioning}

Our results suggest that acute exercise does not improve hippocampal-dependent cognition. By contrast, a large literature in animals and humans shows that long-term exercise over days to weeks can improve performance on a variety of hippocampal-dependent tasks such as spatial memory, contextual fear conditioning, passive avoidance learning, novel object recognition, and pattern separation (Chen et al., 2008; Creer, Romberg, Saksida, van Praag, \& Bussey, 2010; Dery et al., 2013; Falls, Fox, MacAulay, 2010; Fordyce \& Farrar, 1991; Mello, Benetti, Cammarota, \& Izquierdo, 2009; O'Callaghan, Ohle, \& Kelly, 2007; van Praag, 2008). A recent meta-analysis focused on human studies found that acute exercise has moderate to large effects on long-term memory whereas chronic exercise has insignificant effects (Roig et al., 2013), suggesting that different aspects of hippocampal-dependent cognition may be differentially affected by acute and chronic exercise. To our knowledge, few if any studies have examined the effects of acute exercise on cognition in rodents, although acute exercise has been shown to increase both neurotransmitter and neurotrophin content in the hippocampus (Meeusen et al., 2001; Rasmussen et al., 2009). Future research is needed to examine whether different types of hippocampal-dependent cognition are affected by acute exercise in both rodents and humans.

Additionally, our results indicate that acute video watching may impair hippocampal functioning. Previous research has shown that television watching can cause detriments in executive functioning. For example, children that viewed fast-paced television as compared to educational television or drawing showed decreased performance in executive function tasks (Lillard \& Peterson, 2011). This work reveals that television watching may also impair long-term memory in young, healthy adults. While numerous previous acute 
exercise studies have used this type of video watching control group (Coles \& Tomporowski, 2008; Ellemberg \& St-Louis-Deschenes, 2010; Emery et al., 2001), these findings suggest that video watching may not be the optimal control group for studies investigating the effects of acute exercise on cognition.

\section{Beneficial Effects of Acute Exercise Last for up to $2 \mathrm{Hr}$}

To explore how long the exercise-induced cognitive benefits endured, we analyzed the effects of acute exercise on cognition between $30 \mathrm{~min}$ and $2 \mathrm{hr}$ post-exercise. Neither prefrontal cortex- nor hippocampal-dependent cognition displayed a significant three-way interaction between time, intervention type and delay period. This suggests that for either category of cognition, timing of post-exercise testing did not affect the observed cognitive improvement. However, for the prefrontal cortex-dependent tasks, we found a significant two-way interaction between time and intervention type. This suggests that the beneficial effects of acute exercise on prefrontal cortex functioning may persist for up to $2 \mathrm{hr}$ post-exercise cessation. This is one of the first studies to examine how long the cognitive effects of acute exercise persist after exercise. This finding is in line with research showing that acute exercise in both humans and animals produces a transient increase in a variety of neurotransmitters, neuromodulators, and neurotrophins including dopamine, norepinephrine, endogenous opioids, endocannabinoids, brain-derived neurotrophic factor, and insulin-like growth factor 1 (Vivar, Potter, \& van Praag, 2013), with these increases lasting for at least $2 \mathrm{hr}$ after exercise (Chaouloff, 1989; Meeusen et al., 2001; Rasmussen et al., 2009). The fact that these neurochemicals are related to cognition supports the hypothesis that transient increases in their concentrations underlie the observed exercise-induced cognitive enhancements.

As a final point regarding the interaction between exercise and the prefrontal cortex, recent evidence indicates that the medial prefrontal cortex is involved in regulating the motivated behavior of wheel running in rodents (Basso \& Morrell, 2015). Here, we see an example where acute exercise also alters the cognitive functioning of the prefrontal cortex. Thus, exercise appears both to regulate and be regulated by the prefrontal cortex, lending direct experimental support to the concept of a bi-directional relationship between exercise and the brain (Hall \& Fong, 2015; Loprinzi, Herod, Cardinal, \& Noakes, 2013).

\section{ACKNOWLEDGMENTS}

We thank Omar Meziab, Stacey Dasilva, Vincent Dodson, Jonathan Tang, and Samantha Kee for their important early contributions to this work. This work was supported by an NYU ADVANCE grant to NYU for new research ventures. We have no potential conflicts of interest to report. Dr. Basso and Ms. Shang contributed equally to this work.

\section{Supplementary material}

To view supplementary material for this article, please visit http://dx.doi.org/10.1017/S135561771500106X

\section{REFERENCES}

Alloway, T.P., \& Alloway, R.G. (2010). Investigating the predictive roles of working memory and IQ in academic attainment. Journal of Experimental Child Psychology, 106, 20-29.

Alves, C.R., Tessaro, V.H., Teixeira, L.A., Murakava, K., Roschel, H., Gualano, B., \& Takito, M.Y. (2014). Influence of acute highintensity aerobic interval exercise bout on selective attention and short-term memory tasks. Perceptual and Motor Skills, 118, 63-72.

Basso, J.C., \& Morrell, J.I. (2015). The medial prefrontal cortex and nucleus accumbens mediate the motivation for voluntary wheel running in the rat. Behavioral Neuroscience, 129 , 457-472.

Benton, A.L., \& Hamsher, K. (1989). Multilingual Aphasia Examination. Iowa City: AJA Associates.

Boecker, H., Hillman, C.H., Scheef, L., \& Struder, H.K. (Eds) (2012). Functional neuroimaging in exercise and sport sciences. New York: Springer.

Brandt, J., \& Benedict, R.H.B. (2001). Hopkins Verbal Learning Test—Revised. Professional manual. Lutz, FL: Psychological Assessment Resources, Inc.

Brickman, A.M., Stern, Y., \& Small, S.A. (2011). Hippocampal subregions differentially associate with standardized memory tests. Hippocampus, 21, 923-928.

Brisswalter, J., Collardeau, M., \& Rene, A. (2002). Effects of acute physical exercise characteristics on cognitive performance. Sports Medicine, 32, 555-566.

Centofani, C.C. (1975). Selected somatosensory and cognitive test performances as a function of age and education in normal and neurologically impaired adults. Ann Arbor, MI: University of Michigan.

Chaddock, L., Erickson, K.I., Prakash, R.S., Kim, J.S., Voss, M.W., Vanpatter, M., ... Kramer, A.F. (2010). A neuroimaging investigation of the association between aerobic fitness, hippocampal volume, and memory performance in preadolescent children. Brain Research, 1358, 172-183.

Chaddock, L., Erickson, K.I., Prakash, R.S., VanPatter, M., Voss, M.W., Pontifex, M.B., ... Kramer, A.F. (2010). Basal ganglia volume is associated with aerobic fitness in preadolescent children. Developmental Neuroscience, 32, 249-256.

Chang, Y.K., Labban, J.D., Gapin, J.I., \& Etnier, J.L. (2012). The effects of acute exercise on cognitive performance: A metaanalysis. Brain Research, 1453, 87-101.

Chaouloff, F. (1989). Physical exercise and brain monoamines: a review. Acta Physiologica Scandinavica, 137, 1-13.

Chen, H.I., Lin, L.C., Yu, L., Liu, Y.F., Kuo, Y.M., Huang, A.M., ... Jen, C.J. (2008). Treadmill exercise enhances passive avoidance learning in rats: The role of down-regulated serotonin system in the limbic system. Neurobiology of Learning and Memory, 89, 489-496.

Colcombe, S.J., Erickson, K.I., Scalf, P.E., Kim, J.S., Prakash, R., McAuley, E., ... Kramer, A.F. (2006). Aerobic exercise training increases brain volume in aging humans. The Journals of Gerontology. Series A, Biological Sciences and Medical Sciences, 61, 1166-1170. 
Coles, K., \& Tomporowski, P.D. (2008). Effects of acute exercise on executive processing, short-term and long-term memory. Journal of Sports Sciences, 26, 333-344.

Creer, D.J., Romberg, C., Saksida, L.M., van Praag, H., \& Bussey, T.J. (2010). Running enhances spatial pattern separation in mice. Proceedings of the National Academy of Sciences of the United States of America, 107, 2367-2372.

Cutter, G.R., Baier, M.L., Rudick, R.A., Cookfair, D.L., Fischer, J.S., Petkau, J., ... Willoughby, E. (1999). Development of a multiple sclerosis functional composite as a clinical trial outcome measure. Brain, 122(Pt 5), 871-882.

de Jonge, P., \& de Jong, P.F. (1996). Working memory, intelligence and reading ability in children. Personality and Inidividual Differences, 21, 1007-1020.

Dery, N., Pilgrim, M., Gibala, M., Gillen, J., Wojtowicz, J.M., Macqueen, G., \& Becker, S. (2013). Adult hippocampal neurogenesis reduces memory interference in humans: Opposing effects of aerobic exercise and depression. Frontiers in Neuroscience, 7, 66.

Diamond, A. (2013). Executive functions. Annual Review of Psychology, 64, 135-168.

Duncan, G.J., Dowsett, C.J., Claessens, A., Magnuson, K., Huston, A.C., Klebanov, P., ... Japel, C. (2007). School readiness and later achievement. Developmental Psychology, 43, 1428-1446.

Ellemberg, D., \& St-Louis-Deschenes, M. (2010). The effect of acute physical exercise on cognitive function during development. Psychology of Sport and Exercise, 11, 122-126.

Emery, C.F., Honn, V.J., Frid, D.J., Lebowitz, K.R., \& Diaz, P.T. (2001). Acute effects of exercise on cognition in patients with chronic obstructive pulmonary disease. American Journal of Respiratory and Critical Care Medicine, 164, 1624-1627.

Erickson, K.I., Voss, M.W., Prakash, R.S., Basak, C., Szabo, A., Chaddock, L., ... Kramer, A.F. (2011). Exercise training increases size of hippocampus and improves memory. Proceedings of the National Academy of Sciences of the United States of America, 108, 3017-3022.

Etnier, J.L., Salazar, W., Landers, D.M., Petruzzello, S.J., Han, M., \& Nowell, P. (1997). The influence of physical fitness and exercise upon cognitive functioning: A meta-analysis. Journal of Sport \& Exercise Psychology, 19, 249-277.

Falls, W.A., Fox, J.H., \& MacAulay, C.M. (2010). Voluntary exercise improves both learning and consolidation of cued conditioned fear in C57 mice. Behavioural Brain Research, 207, 321-331.

Fordyce, D.E., \& Farrar, R.P. (1991). Enhancement of spatial learning in F344 rats by physical activity and related learningassociated alterations in hippocampal and cortical cholinergic functioning. Behavioural Brain Research, 46, 123-133.

Gabbard, C., \& Barton, J. (1979). Effects of physical activity on mathematical computation among young children. The Journal of Psychology, 103, 287-288.

Gathercole, S.E., Alloway, T.P., Willis, C., \& Adams, A.M. (2006). Working memory in children with reading disabilities. Journal of Experimental Child Psychology, 93, 265-281.

Golden, C.J. (1975). A group version of the Stroop Color and Word Test. Journal of Personality Assessment, 39, 386-388.

Golden, C.J. (1978). Stroop color and word test. Illinois: Stoelting Company.

Greenwood, B.N., \& Fleshner, M. (2011). Exercise, stress resistance, and central serotonergic systems. Exercise and Sport Sciences Reviews, 39, 140-149.
Guiney, H., \& Machado, L. (2013). Benefits of regular aerobic exercise for executive functioning in healthy populations. Psychonomic Bulletin \& Review, 20, 73-86.

Hall, P.A., \& Fong, G.T. (2015). Temporal self-regulation theory: A neurobiologically informed model for physical activity behavior. Frontiers in Human Neuroscience, 9, 117.

Hillman, C.H., Kamijo, K., \& Scudder, M. (2011). A review of chronic and acute physical activity participation on neuroelectric measures of brain health and cognition during childhood. Preventive Medicine, 52(Suppl 1), S21-S28.

Hofmann, W., Schmeichel, B.J., \& Baddeley, A.D. (2012). Executive functions and self-regulation. Trends in Cognitive Sciences, 16, 174-180.

Hopkins, M.E., Davis, F.C., Vantieghem, M.R., Whalen, P.J., \& Bucci, D.J. (2012). Differential effects of acute and regular physical exercise on cognition and affect. Neuroscience, 215, 59-68.

Howie, E.K., Schatz, J., \& Pate, R.R. (2015). Acute effects of classroom exercise breaks on executive function and math performance: A dose-response study. Research Quarterly for Exercise and Sport, 86, 217-224.

Hsu, N.S., Novick, J.M., \& Jaeggi, S.M. (2014). The development and malleability of executive control abilities. Frontiers in Behavioral Neuroscience, 8, 221.

Kronenberg, G., Bick-Sander, A., Bunk, E., Wolf, C., Ehninger, D., \& Kempermann, G. (2006). Physical exercise prevents agerelated decline in precursor cell activity in the mouse dentate gyrus. Neurobiology of Aging, 27, 1505-1513.

Lambourne, K., \& Tomporowski, P. (2010). The effect of exercise-induced arousal on cognitive task performance: A metaregression analysis. Brain Research, 1341, 12-24.

Lillard, A.S., \& Peterson, J. (2011). The immediate impact of different types of television on young children's executive function. Pediatrics, 128, 644-649.

Loprinzi, P.D., Herod, S.M., Cardinal, B.J., \& Noakes, T.D. (2013). Physical activity and the brain: A review of this dynamic, bi-directional relationship. Brain Research, 1539, 95-104.

Loprinzi, P.D., \& Kane, C.J. (2015). Exercise and cognitive function: A randomized controlled trial examining acute exercise and free-living physical activity and sedentary effects. Mayo Clinic Proceedings, 90, 450-460.

McMorris, T.G., \& Graydon, J. (2000). The effect of incremental exercise on cognitive performance. International Journal of Sport Psychology, 31, 66-81.

McNaughten, D., \& Gabbard, C. (1993). Physical exertion and immediate mental performance of sixth-grade children. Perceptual and Motor Skills, 77, 1155-1159.

Meeusen, R. (2005). Exercise and the brain: Insight in new therapeutic modalities. Annals of transplantation, 10, 49-51.

Meeusen, R., Piacentini, M.F., \& De Meirleir, K. (2001). Brain microdialysis in exercise research. Sports Medicine, 31, 965-983.

Mello, P.B., Benetti, F., Cammarota, M., \& Izquierdo, I. (2009). Physical exercise can reverse the deficit in fear memory induced by maternal deprivation. Neurobiology of Learning and Memory, 92, 364-369.

Nanda, B., Balde, J., \& Manjunatha, S. (2013). The acute effects of a single bout of moderate-intensity aerobic exercise on cognitive functions in healthy adult males. Journal of Clinical and Diagnostic Research, 7, 1883-1885.

Netz, Y., Tomer, R., Axelrad, S., Argov, E., \& Inbar, O. (2007). The effect of a single aerobic training session on cognitive flexibility in late middle-aged adults. International Journal of Sports Medicine, 28, 82-87. 
Novick, J.M., Trueswell, J.C., \& Thompson-Schill, S.L. (2005). Cognitive control and parsing: Reexamining the role of Broca's area in sentence comprehension. Cognitive, Affective \& Behavioral Neuroscience, 5, 263-281.

O’Callaghan, R.M., Ohle, R., \& Kelly, A.M. (2007). The effects of forced exercise on hippocampal plasticity in the rat: A comparison of LTP, spatial- and non-spatial learning. Behavioural Brain Research, 176, 362-366.

Passolunghi, M.C., \& Siegel, L.S. (2001). Short-term memory, working memory, and inhibitory control in children with difficulties in arithmetic problem solving. Journal of Experimental Child Psychology, 80, 44-57.

Pennington, B.F., Moon, J., Edgin, J., Stedron, J., \& Nadel, L. (2003). The neuropsychology of Down syndrome: Evidence for hippocampal dysfunction. Child Development, 74, 75-93.

Rasmussen, P., Brassard, P., Adser, H., Pedersen, M.V., Leick, L., Hart, E., ... Pilegaard, H. (2009). Evidence for a release of brainderived neurotrophic factor from the brain during exercise. Experimental Physiology, 94, 1062-1069.

Roig, M., Nordbrandt, S., Geertsen, S.S., \& Nielsen, J.B. (2013). The effects of cardiovascular exercise on human memory: A review with meta-analysis. Neuroscience and Biobehavioral Reviews, 37, 1645-1666.

Ruff, R.M., Light, R.H., Parker, S.B., \& Levin, H.S. (1996). Benton Controlled Oral Word Association Test: Reliability and updated norms. Archives of Clinical Neuropsychology, 11, 329-338.

Shah, P., \& Miyake, A. (1999). Toward unified theories of working memory: Emerging general consensus, unresolved theoretical issues, and future research directions. In A. Miyake, \& P. Shah (Eds.), Models of working memory: Mechanisms of active maintenance and executive control (pp. 442-482). New York: Oxford University Press.

Smith, A. (1991). Symbol Digit Modalities Test. Los Angeles, CA: Western Psychological Services.
Tombaugh, T.N. (2004). Trail Making Test A and B: Normative data stratified by age and education. Archives of Clinical Neuropsychology, 19, 203-214.

Tombaugh, T.N., Kozak, J., \& Rees, L. (1999). Normative data stratified by age and education for two measures of verbal fluency: FAS and animal naming. Archives of Clinical Neuropsychology, 14, 167-177.

Tomporowski, P.D. (2003). Effects of acute bouts of exercise on cognition. Acta Psychologica, 112, 297-324.

Tomporowski, P.D., \& Ellis, N.M. (1986). Effects of exercise on cognitive processes: A review. Psychological Bulletin, 99, 338-346.

van Praag, H. (2008). Neurogenesis and exercise: Past and future directions. Neuromolecular Medicine, 10, 128-140.

Verstynen, T.D., Lynch, B., Miller, D.L., Voss, M.W., Prakash, R.S., Chaddock, L., ... Erickson, K.I. (2012). Caudate Nucleus Volume Mediates the Link between Cardiorespiratory Fitness and Cognitive Flexibility in Older Adults. Journal of Aging Research, 2012, 939285.

Vivar, C., Potter, M.C., \& van Praag, H. (2013). All about running: Synaptic plasticity, growth factors and adult hippocampal neurogenesis. Current Topics in Behavioral Neurosciences, 15, 189-210.

Voss, M.W., Vivar, C., Kramer, A.F., \& van Praag, H. (2013). Bridging animal and human models of exercise-induced brain plasticity. Trends in Cognitive Sciences, 17, 525-544.

Wang, C.C., Shih, C.H., Pesce, C., Song, T.F., Hung, T.M., \& Chang, Y.K. (2015). Failure to identify an acute exercise effect on executive function assessed by the Wisconsin Card Sorting Test. Journal of Sport and Health Sciecne, 4, 64-72.

Wechsler, D. (1981). WAIS-R manual: Wechsler adult intelligence scale-revised. San Antonio, TX: Psychological Corporation.

Wechsler, D. (2008). Wechsler Adult Intelligence Scale-Fourth Edition. San Antonio, TX: The Psychological Corporation.

Yeudall, L.T., Reddon, J.R., Gill, D.M., \& Stefanyk, W.O. (1987). Normative data for the Halstead-Reitan neuropsychological tests stratified by age and sex. Journal of Clinical Psychology, 43, 346-367. 